\title{
Additional utility of insiders with imperfect dynamical information
}

\author{
José M. Corcuera \\ Universitat de Barcelona, Facultat de Matemàtiques, Gran Via de les Corts \\ Catalanes 585, 08007 Barcelona, Spain \\ Peter Imkeller \\ Humboldt-Universität zu Berlin, Institut für Mathematik, \\ Unter den Linden 6, 10999 Berlin, Germany \\ Arturo Kohatsu-Higa \\ Universitat Pompeu Fabra, Department of Economics \\ and Business, Ramón Trias Fargas 25-27, 08005 Barcelona, Spain \\ David Nualart \\ Universitat de Barcelona, Facultat de Matemàtiques, Gran Via de les Corts \\ Catalanes 585, 08007 Barcelona, Spain
}

April 4, 2003 


\begin{abstract}
In this paper we consider an insider with privileged information that is affected by an independent noise vanishing as the revelation time approaches. At this time, information is available to every trader. Our financial markets are based on Wiener space.

In probabilistic terms we obtain an infinite dimensional extension of Jacod's theorem to cover cases of progressive enlargement of filtrations. The application of this result gives the semimartingale decomposition of the original Wiener process under the progressively enlarged filtration.

As an application we prove that if the rate at which the additional noise in the insider's information vanishes is slow enough then there is no arbitrage and the additional utility of the insider is finite.
\end{abstract}

JEL: G140

Keywords: Insider trading; Enlargement of filtrations; Malliavin's calculus; Utility maximization; Arbitrage 


\section{Introduction}

Financial markets inherently have asymmetry of information. That is, there are different types of traders whose behavior is induced by different types of information they possess (or not). In the classical setting for financial markets one assumes that all traders share the same information which allows the study of non-arbitrage and equilibrium conditions. In this article we are interested in finding settings where a continuous time financial market can accept continuous differences in information between traders. These differences can disappear at certain revelation times. Our conclusion is that one can construct such markets and still achieve non-arbitrage under some restrictions on the trading strategies of the informed agents.

One of the aspects of this complex problem is to study the effects of changes in information of different agents. One can find literature in mathematical economics as well as in stochastic process theory dealing with problems of this nature. In the latter area the most frequently used techniques are based on the enlargement of filtrations. A basic reference on this topic is the series of papers in the Séminaire de Calcul Stochastique (1982/83) of the University Paris VI published in 1985.

In the past few years we have seen expanding interest in this area. Articles where applications of the enlargement of filtrations technique is used to portfolio optimization of an insider are Karatzas and Pikovsky (1996), Imkeller (1996 ,1997), Amendinger et al. (1998), Grorud and Pontier (1998) and Imkeller et al. (2001). The set up in most of these works is to consider two small agents who optimize their logarithmic utility. One considers the differential of utility between these two agents supposing that one of the agents is better informed than the other. One of the important conclusions of this body of work is that if the differential is generated by the initial knowledge of the value of a random variable then the additional utility is the relative entropy of this random variable with respect to the original probability measure, see for instance remark 2.5 in Karatzas and Pikovsky (1996).

In most of these results the extra information of the insider can be classified into two types. In the first, the insider has direct access to the price of the underlying at some time in the future $T$. In this case the utility differential is infinity and there is arbitrage which is realized at this future time $T$. In the second, the insider knows the price of the underlying perturbed by a remaining independent noise which is alive through the life of the trades. 
In this case the additional utility is finite due to the extra component of noise. Nevertheless we encounter the somewhat odd situation that this extra component of noise does not vanish even at the revelation time $T$.

In this sense all the previous results assume that the knowledge of the additional information does not change through time. In mathematical terms this amounts to dealing with an initial enlargement of filtrations by a random variable. Related problems are often solved using some variant of a well known theorem by Jacod, see Jacod (1985), and Grorud and Pontier (1998) for a discussion of different conditions under which Jacod's result holds true. In this article we propose an infinite dimensional extension of this theorem to cover cases of progressive enlargement of filtrations. This, in particular, covers situations in which the differential of information may be a different random variable at each time, but this difference is generated by an independent stochastic process.

Most importantly, we consider the following type of example: a trader with additional information consisting of the price at final time with some blurring additional noise. This noise dynamically tends to 0 as final time gets closer. So we deal with a trader whose additional information is continuously getting clearer to him as time evolves.

As already mentioned, in other relevant papers, see section 3 in Karatzas and Pikovsky (1996) and 4.3 in Amendinger et al. (1998), this amount of blurring noise is fixed throughout the trading interval.

Other approaches to insider's effects in financial markets are made by Kyle (1985), and Back (1992) in the context of an equilibrium theory, with different kind of traders acting in the market, and by Baudoin (2001), where the true model of stock prices is partially observed and where the insider's extra information consists of the the law of some functional of the future prices of stocks. Even though, in this latter case, enlargement of filtrations techniques are not relevant due to the type of additional information being considered, the author establishes a deep relationship with the case where the extra information is the value of a functional of the future prices of stocks.

The results in our cornerstone examples state that if the rate at which the blurring noise disappears is slow enough then there will be a finite additional logarithmic utility and possibly no arbitrage.

This situation corresponds to a more natural situation where the information retained by the insider is improving as times evolves. The minimum rate at which the blurring has to go to zero in order to achieve no-arbitrage could also be interpreted as the necessary noise that has to be generated by 
noise traders, see Back (1992), in order not to reveal the information the insider possesses.

We corroborate the above results also for the case where the additional information is the maximum of the value of the stock plus some blurring noise. Technically this case is somewhat different from the previous one since the arbitrage does not come from the behavior of the optimal strategies at revelation time but as a consequence of the random time at which the maximum is reached. Nevertheless the same conclusions as in the previous example are valid here.

We consider financial markets driven by a Wiener process and we use Malliavin's Calculus to obtain the formula of the compensator, or the information drift, generating a Wiener process in the enlarged filtration.

Another important difference with respect to previous papers is that we work with different hypotheses about the random variables which constitute the additional information. These hypotheses are easy to check using Malliavin's Calculus.

For simplicity, we restrict our analysis to the case of a one-dimensional model, although our approach can be easily extended to a multidimensional framework.

The paper is organized in the following way. Section 2 is devoted to finding a formula for the compensator of the Wiener process. In Section 3 we apply the previous results to several basic examples, while Section 4 deals with the analyzing the effects of the information drift in the additional logarithmic utility and possibilities of arbitrage.

\section{An infinite dimensional extension of Jacod's Theorem}

Consider a Wiener process $W=\left\{W_{t}, 0 \leq t \leq T\right\}$ defined on a complete probability space $(\Omega, \mathcal{F}, P)$. We denote by $\left(\mathcal{F}_{t}\right)_{t \in[0, T]}$ the natural filtration generated by the Wiener process and the sets of $P$-measure zero.

Assume that the additional information until time $t$ is given by a family of random variables $\left\{L_{s}, s \leq t\right\}$. Suppose that these random variables have the following structure:

$$
L_{t}=G\left(X, Y_{t}\right)
$$


where $X$ is an $\mathcal{F}_{T}$-measurable random variable, the process $Y=\left\{Y_{t}, 0 \leq t \leq\right.$ $T\}$ is independent of the $\sigma$-algebra $\mathcal{F}_{T}$, and $G: \mathbb{R}^{2} \rightarrow \mathbb{R}$ is a given measurable function. In that sense we can define $\mathcal{H}_{t}$ as the usual augmentation of the filtration $\mathcal{F}_{t} \vee \sigma\left(L_{s}, s \leq t\right.$ ) (see Rogers and Williams (1979)).

The filtration $\left(\mathcal{F}_{t}\right)_{t \in[0, T]}$ gives the regular trader's evolution of knowledge, whereas the enlarged filtration $\left(\mathcal{H}_{t}\right)_{t \in[0, T]}$ describes the insider's filtration. The random variable $X$ contains the additional information available to the privileged trader, and the random variables $Y_{t}$ represent an additional noise that perturbs this additional information. Therefore one expects in general that $Y_{T}=0$ and that the variance of the noise should decrease to zero as the revelation time $T$ approaches.

For each $t \in[0, T]$, we denote by $P_{t}(\omega, d x)$ a regular version of the conditional law of a random variable $X$ given the $\sigma$-field $\mathcal{F}_{t}$, abbreviating it sometimes by $P_{t}(d x)$. We can choose this version in such a way that the following conditions are satisfied:

1. For every Borel set $B$ on the real line, $\left\{P_{t}(B), t \in[0, T]\right\}$ is an $\mathcal{F}_{t^{-}}$ progressively measurable process.

2. For every $(t, \omega) \in[0, T] \times \Omega, P_{t}(\omega, d x)$ is a probability measure on the real line.

3. For any bounded and $\mathcal{F}_{t}$-adapted process $u$ and for any bounded and measurable function $f$ on $\mathbb{R}$, we have

$$
E\left(f(X) \int_{0}^{T} u_{t} d t\right)=E \int_{0}^{T} u_{t}\left(\int_{\mathbb{R}} f(x) P_{t}(d x)\right) d t .
$$

In order to establish the general formula for the compensator, we require the random variable $X$ to belong to a certain class $\mathcal{L}_{1}$ to be defined below.

Definition 1 We say that an $\mathcal{F}_{T}$-measurable random variable $X$ belongs to the class $\mathcal{L}_{1}$ if there exists a random kernel $P_{t}^{(1)}(\omega, d x)$ such that

1. For every Borel set $B$ in the real line, $\left\{P_{t}^{(1)}(B), t \in[0, T)\right\}$ is an $\mathcal{F}_{t^{-}}$ progressively measurable process.

2. For every $(t, \omega) \in[0, T) \times \Omega, P_{t}^{(1)}(\omega, d x)$ is a signed measure on the real line. 
3. For every $t \in[0, T), E \int_{0}^{t}\left|P_{t}^{(1)}\right| d t<\infty$, where $\left|P_{t}^{(1)}\right|(\omega)$ denotes the total variation of the measure $P_{t}^{(1)}(\omega, \cdot)$.

4. For any bounded and $\mathcal{F}_{t}$-adapted process $u$, for any bounded and measurable function $f$ on $\mathbb{R}$, and for every $t \in[0, T)$, we have

$$
E\left(f(X) \int_{0}^{t} u_{t} d W_{t}\right)=E\left(\int_{0}^{t} u_{t}\left(\int_{\mathbb{R}} f(x) P_{t}^{(1)}(d x)\right) d t\right) .
$$

In order to understand the above condition better, let $X$ be an arbitrary $\mathcal{F}_{T}$-measurable random variable. For any measurable and bounded function $f$ on $\mathbb{R}$ we denote by $\Phi^{f}=\left\{\Phi_{t}^{f}, t \in[0, T]\right\}$ the $\mathcal{F}_{t}$-predictable process appearing in the Ito stochastic integral representation of $f(X)$ :

$$
f(X)=E(f(X))+\int_{0}^{T} \Phi_{t}^{f} d W_{t}
$$

Therefore in order to construct the measure $P_{t}^{(1)}(d x)$ in the above definition one may use that

$$
E\left(f(X) \int_{0}^{T} u_{t} d W_{t}\right)=E\left(\int_{0}^{T} u_{t} \Phi_{t}^{f} d t\right)
$$

Therefore the problem may be solved if we can construct an adapted signed measure $P_{t}^{(1)}$ such that

$$
\Phi_{t}^{f}=\int_{\mathbb{R}} f(x) P_{t}^{(1)}(d x)
$$

for almost all $(t, \omega)$. At first, it may seem that the signed measure can be defined using simple functions $f$ and then expanding the defined measure to the whole sample space if we have some continuity property. That is, if $f_{n}$ is a sequence of measurable and uniformly bounded functions which converge pointwise to $f$, then

$$
\lim _{n \rightarrow \infty} E \int_{0}^{T}\left|\Phi_{t}^{f_{n}}-\Phi_{t}^{f}\right|^{2} d t=0 .
$$

Nevertheless, this continuity property does not imply directly the existence of the kernel $P_{t}^{(1)}(d x)$. 
A different approach to show the existence of this kernel is to use ClarkOcone's formula, see for instance Nualart (1995), and deduce directly from there the definition of the measure $P_{t}^{(1)}$. In fact we establish the following proposition.

Proposition 2 Suppose that there exists a (localization) process $h:[0, T] \times$ $\Omega \rightarrow \mathbb{R}$ such that $X \in \mathbb{D}^{1,2}$ is such that for almost all $(t, \omega) \in[0, T] \times \Omega$, $\int_{t}^{T} h(l) D_{l} X d l=0$ implies $h(s) D_{t} X=0, s \in[0, T]$ and the process

$$
\frac{h(s) D_{t} X}{\int_{t}^{T} h(l) D_{l} X d l} \mathbf{1}_{[t, T]}(s)
$$

belongs to the domain of the divergence (here we define the quotient as 0 if the denominator is 0). Assume also that

$$
\int_{0}^{T} E\left(\left|\int_{t}^{T} \frac{h(s) D_{t} X}{\int_{t}^{T} h(l) D_{l} X d l} d W_{s}\right| \mid \mathcal{F}_{t}\right) d t<\infty .
$$

Then $X$ belongs to $\mathcal{L}_{1}$ and

$$
P_{t}^{(1)}(B)=E\left(\mathbf{1}_{B}(X) \int_{t}^{T} \frac{h(s) D_{t} X}{\int_{t}^{T} h(l) D_{l} X d l} d W_{s} \mid \mathcal{F}_{t}\right) .
$$

Proof. Fixed $f \in C_{b}^{1}$ the Clark-Ocone formula for random variables $X \in \mathbb{D}^{1,2}$ states that

$$
f(X)=E(f(X))+\int_{0}^{T} E\left[D_{t}(f(X)) \mid \mathcal{F}_{t}\right] d W(t) .
$$

Then, for any bounded $\mathcal{F}_{t^{-}}$adapted process $u$,

$$
\begin{aligned}
E\left(f(X) \int_{0}^{T} u_{t} d W_{t}\right) & =E \int_{0}^{T} u_{t} E\left[D_{t}(f(X)) \mid \mathcal{F}_{t}\right] d t=E \int_{0}^{T} u_{t} D_{t}(f(X)) d t \\
& =\int_{0}^{T} E\left(u_{t} D_{t} X \int_{t}^{T} h(s) \frac{D_{s}(f(X))}{\int_{t}^{T} h(r) D_{r} X d r} d s\right) d t \\
& =\int_{0}^{T} E\left(\int_{t}^{T} \frac{h(s) D_{t} X D_{s}\left(u_{t} f(X)\right)}{\int_{t}^{T} h(r) D_{r} X d r} d s\right) d t \\
& =E \int_{0}^{T} u_{t} E\left(f(X) \int_{t}^{T} \frac{h(s) D_{t} X}{\int_{t}^{T} h(r) D_{r} X d r} d W_{s} \mid \mathcal{F}_{t}\right) d t .
\end{aligned}
$$


The result can be extended to any bounded and measurable function $f$ by an argument of approximation. Moreover, (1) implies condition 3. in the definition of $\mathcal{L}_{1}$.

Localizations can be useful when the random variable $X$ has undesirable properties in certain smooth subsets of $\Omega$. For more details on this, see Nualart (1995).

In Imkeller et al. (2001), the authors have developed a Malliavin Calculus for measure-valued random variables and have introduced $P_{t}^{(1)}(d x)$ as the kernel appearing in the Clark-Ocone stochastic integral representation of the measure-valued random variable $\delta_{X(\omega)}$. More precisely, suppose that $\delta_{X}$ belongs to $\mathbb{D}^{1,2}(M)$, where $M$ is the space of signed measures on the real line, equipped with the total variation norm. Then, Proposition A.1 of Imkeller et al. (2001) states that $X$ belongs to the class $\mathcal{L}_{1}$, and

$$
P_{t}^{(1)}(d x)=E\left(D_{t}\left[\delta_{X}\right] \mid \mathcal{F}_{t}\right)(d x) .
$$

The following proposition is a generalization of Theorem 2.1 in Imkeller et al. (2001).

Proposition 3 Suppose that $X$ is an $\mathcal{F}_{T}$-measurable random variable in the class $\mathcal{L}_{1}$. Assume that for almost all $(t, \omega)$, the signed measure $P_{t}^{(1)}(d x)$ is absolutely continuous with respect to $P_{t}(d x)$, and set

$$
\alpha_{t}(x)=\frac{P_{t}^{(1)}(d x)}{P_{t}(d x)} .
$$

We can choose a version of $\alpha_{t}(x)$ which is $\mathcal{P} \otimes \mathcal{B}(\mathbb{R})$-measurable, where $\mathcal{P}$ denotes the $\mathcal{F}_{t}$-progressive $\sigma$-field. Set

$$
\beta_{t}=E\left(\alpha_{t}(X) \mid \mathcal{H}_{t}\right),
$$

where we consider a progressively measurable version. Assume that $\int_{0}^{T}\left|\beta_{s}\right| d s<$ $\infty$ a.s., then $W_{t}-\int_{0}^{t} \beta_{s} d s$ is a Wiener process with respect to the filtration $\left(\mathcal{H}_{t}\right)_{t \in[0, T]}$.

Proof. We can choose a version of $\alpha_{t}(x)$ which is $\mathcal{P} \otimes \mathcal{B}(\mathbb{R})$-measurable since any adapted process has a version that is progressively measurable, see Meyer (1996). Let $A \in \mathcal{F}_{s}, h$ a bounded measurable function on $\mathbb{R}^{n}$, and 
$s_{1} \leq \cdots \leq s_{n} \leq s<t$. Set $H=h\left(L_{s_{1}}, \ldots, L_{s_{n}}\right)$. Denote $Y=\left(Y_{s_{1}}, \ldots, Y_{s_{n}}\right)$ and $E_{Y}(\cdot)$ the conditional expectation fixed $Y=\left(y_{s_{1}}, \ldots, y_{s_{n}}\right)$. Then we have

$$
\begin{aligned}
& E_{Y}\left(\left(W_{t}-W_{s}\right) \mathbf{1}_{A} H\right) \\
= & E\left(\mathbf{1}_{A}\left(W_{t}-W_{s}\right) h\left(G\left(X, y_{s_{1}}\right), \ldots, G\left(X, y_{s_{n}}\right)\right)\right) \\
= & E\left(\mathbf{1}_{A} \int_{s}^{t} \int_{\mathbb{R}} h\left(G\left(x, y_{s_{1}}\right), \ldots, G\left(x, y_{s_{n}}\right)\right) P_{u}^{(1)}(d x) d u\right) \\
= & E\left(\mathbf{1}_{A} \int_{s}^{t} \int_{\mathbb{R}} h\left(G\left(x, y_{s_{1}}\right), \ldots, G\left(x, y_{s_{n}}\right)\right) \alpha_{u}(x) P_{u}(d x) d u\right) \\
= & E\left(\mathbf{1}_{A} \int_{s}^{t} h\left(G\left(X, y_{s_{1}}\right), \ldots, G\left(X, y_{s_{n}}\right)\right) \alpha_{u}(X) d u\right) \\
= & E_{Y}\left(\mathbf{1}_{A} H \int_{s}^{t} \alpha_{u}(X) d u\right) .
\end{aligned}
$$

If we take expectation with respect to $Y$ we obtain

$$
\begin{aligned}
& E\left(\left(W_{t}-W_{s}\right) \mathbf{1}_{A} H\right) \\
= & E\left(\mathbf{1}_{A} H \int_{s}^{t} \alpha_{u}(X) d u\right) \\
= & E\left(\mathbf{1}_{A} H \int_{s}^{t} E\left(\alpha_{u}(X) \mid \mathcal{H}_{u}\right) d u\right) .
\end{aligned}
$$

Therefore $W_{t}-\int_{0}^{t} \beta_{s} d s$ is a continuous local martingale in the filtration $\left(\mathcal{H}_{t}\right)_{t \in[0, T[}$. Lévy's characterization theorem and the condition $\int_{0}^{T}\left|\beta_{s}\right| d s<\infty$ a.s. imply the result.

This approach using random kernels and their absolute continuity is similar to that of Yor (1995). As a corollary of the above proposition one also obtains the classical version of the Theorem by Jacod.

Corollary 4 Denote by $P^{X}$ the law of the random variable $X \in \mathcal{F}_{T}$. Assume that $P_{t} \ll P^{X}$ for any $t \in[0, T)$ a.s., let

$$
\begin{aligned}
p_{t}(x)(\omega) & =\frac{d P_{t}}{d P^{X}}(\omega, x) \\
\alpha_{t}(x) & =\frac{\frac{d}{d t}<p \cdot(x), W>_{t}}{p_{t}(x)} .
\end{aligned}
$$


Suppose that $E \int_{0}^{t}\left|\alpha_{s}(X)\right| d s<\infty$ for any $t \in[0, T)$ and that $\int_{0}^{T} E\left(\mid \alpha_{t}(X) \| \mathcal{H}_{t}\right) d t<$ $\infty$ a.s . Then $W_{t}-\int_{0}^{t} E\left(\alpha_{s}(X) \mid \mathcal{H}_{t}\right) d s, 0 \leq t<T$ is a Wiener process with respect to the filtration $\left(\mathcal{H}_{t}\right)$ on $[0, T]$.

Proof. It is known that $p_{t}(x)=\frac{d P_{t}}{d P^{X}}, t \in[0, T)$, is a continuous local martingale. Then, we have

$$
\begin{aligned}
E\left(f(X) \int_{0}^{t} u(s) d W_{s}\right) & =E\left(\int_{\mathbb{R}} f(x) \frac{d P_{t}}{d P^{X}}(x)\left(\int_{0}^{t} u(s) d W_{s}\right) P^{X}(d x)\right) \\
& =E\left(\int_{\mathbb{R}} f(x) \int_{0}^{t} u(s) d<p .(x), W>_{s} P^{X}(d x)\right) .
\end{aligned}
$$

From here it follows that

$$
P_{t}^{(1)}(d x)=\frac{d}{d t}<p .(x), W>_{t} P^{X}(d x)
$$

Notice that $P\left(p_{t}(x)=0\right)=0$ :

$$
\begin{aligned}
P\left(p_{t}(x)\right. & =0)=E\left(\mathbf{1}_{\left\{p_{t}(x)=0\right\}}\right)=E\left(E\left(\mathbf{1}_{\left\{p_{t}(x)=0\right\}} \mid \mathcal{F}_{t}\right)\right) \\
& =E\left(\int_{\left\{p_{t}(x)=0\right\}} p_{t}(x) P^{X}(d x)\right)=0 .
\end{aligned}
$$

As a consequence, $\alpha_{t}(x)$ is well defined and it coincides with the RadonNikodym density of $P_{t}^{(1)}(d x)$ with respect to $P_{t}(d x)$. Therefore the assumptions of the previous proposition are satisfied and we obtain the conclusion.

Notice that Jacod's theorem was established without the condition $E \int_{0}^{t}\left|\alpha_{s}(X)\right| d s<\infty, t \in[0, T)$ (see Jacod (1985)). In fact the theorem was proved by assuming only that $\int_{0}^{T}\left|\alpha_{t}(X)\right| d t<\infty$ a.s. Nevertheless we can prove an infinite dimensional extension of Jacod's Theorem in a direct way.

Theorem 5 Denote by $P^{X}$ the law of the random variable $X \in \mathcal{F}_{T}$. Assume that $P_{t} \ll P^{X}$ for any $t \in[0, T)$ a.s., let

$$
\begin{aligned}
p_{t}(x)(\omega) & =\frac{d P_{t}}{d P^{X}}(\omega, x) \\
\alpha_{t}(x) & =\frac{\frac{d}{d t}<p \cdot(x), W>_{t}}{p_{t}(x)} .
\end{aligned}
$$


Suppose that $E\left(\left|\alpha_{t}(X)\right|\right)<\infty$ for every $t \in[0, T)$, and, setting $\beta_{t}=$ $E\left(\alpha_{t}(X) \mid \mathcal{H}_{t}\right)$, that $\int_{0}^{T}\left|\beta_{s}\right| d s<\infty$ a.s. Then $W_{t}-\int_{0}^{t} \beta_{s} d s$ is a Wiener process with respect to the filtration $\left(\mathcal{H}_{t}\right)$ on $[0, T]$.

Proof. Let $A \in \mathcal{F}_{s}, \quad h$ a bounded measurable function on $\mathbb{R}^{n}$, and $s_{1} \leq \cdots \leq s_{n} \leq s<t$. Set $H=h\left(L_{s_{1}}, \ldots, L_{s_{n}}\right)$. Denote $Y=\left(Y_{s_{1}}, \ldots, Y_{s_{n}}\right)$ and $E_{Y}(\cdot)$ the conditional expectation fixed $Y=\left(y_{s_{1}}, \ldots, y_{s_{n}}\right)$. Let $\tau_{n}^{p(x)}=$ $\inf \left\{t, p_{t}(x) \geq n\right\}$. Note that for $n \in \mathbb{N}, \tau_{n}^{p(\cdot)}$ is measurable in $(\omega, x)$. Then we have

$$
\begin{aligned}
& E_{Y}\left(\left(W_{t \wedge \tau_{n}^{p(X)}}-W_{s \wedge \tau_{n}^{p(X)}}\right) \mathbf{1}_{A} H\right) \\
= & E\left(\mathbf{1}_{A}\left(W_{t \wedge \tau_{n}^{p(X)}}-W_{s \wedge \tau_{n}^{p(X)}}\right) h\left(G\left(X, y_{s_{1}}\right), \ldots, G\left(X, y_{s_{n}}\right)\right)\right) \\
= & E\left(\mathbf{1}_{A} \int_{\mathbb{R}} p_{t}(x)\left(W_{t \wedge \tau_{n}^{p(x)}}-W_{s \wedge \tau_{n}^{p(x)}}\right) h\left(G\left(x, y_{s_{1}}\right), \ldots, G\left(x, y_{s_{n}}\right)\right) P^{X}(d x)\right) \\
= & \int_{\mathbb{R}} h\left(G\left(x, y_{s_{1}}\right), \ldots, G\left(x, y_{s_{n}}\right)\right) E\left(\mathbf{1}_{A} p_{t}(x)\left(W_{t \wedge \tau_{n}^{p(x)}}-W_{s \wedge \tau_{n}^{p(x)}}\right)\right) P^{X}(d x) \\
= & \int_{\mathbb{R}} h\left(G\left(x, y_{s_{1}}\right), \ldots, G\left(x, y_{s_{n}}\right)\right) E\left(\mathbf{1}_{A} E\left(p_{t}(x)\left(W_{t \wedge \tau_{n}^{p(x)}}-W_{\left.s \wedge \tau_{n}^{p(x)}\right)}\right) \mid \mathcal{F}_{s}\right)\right) P^{X}(d x) \\
= & \int_{\mathbb{R}} h\left(G\left(x, y_{s_{1}}\right), \ldots, G\left(x, y_{s_{n}}\right)\right) E\left(\mathbf{1}_{A} \int_{s \wedge \tau_{n}^{p(x)}}^{t \wedge \tau_{n}^{p(x)}}<p .(x), W>_{u} d u\right) P^{X}(d x)
\end{aligned}
$$




$$
\begin{aligned}
& =E\left(\mathbf{1}_{A} \int_{\mathbb{R}} h\left(G\left(x, y_{s_{1}}\right), \ldots, G\left(x, y_{s_{n}}\right)\right) \int_{s \wedge \tau_{n}^{p(x)}}^{t \wedge \tau_{n}^{p(x)}}<p \cdot(x), W>_{u} d u\right) P^{X}(d x) \\
& =E\left(\mathbf{1}_{A}\left(\int_{s}^{t} \int_{\mathbb{R}} h\left(G\left(x, y_{s_{1}}\right), \ldots, G\left(x, y_{s_{n}}\right)\right) \mathbf{1}_{\left[0, \tau_{n}^{p(x)}\right]}(u)<p \cdot(x), W>_{u} P^{X}(d x)\right) d u\right) \\
& =\int_{s}^{t} E\left(\mathbf{1}_{A} \int_{\mathbb{R}} h\left(G\left(x, y_{s_{1}}\right), \ldots, G\left(x, y_{s_{n}}\right)\right) \mathbf{1}_{\left[0, \tau_{n}^{p(x)}\right]}(u) \frac{<p \cdot(x), W>_{u}}{p_{u}(x)} P_{u}(d x)\right) d u \\
& =\int_{s}^{t} E\left(E\left(\left.\mathbf{1}_{A} h\left(G\left(x, y_{s_{1}}\right), \ldots, G\left(x, y_{s_{n}}\right) \mathbf{1}_{\left[0, \tau_{n}^{p(x)}\right]}(u) \frac{<p \cdot(x), W>_{u}}{p_{u}(x)} \mid \mathcal{F}_{u}\right)\right|_{x=X}\right) d u\right. \\
& =\int_{s}^{t} E\left(\mathbf{1}_{A} h\left(G\left(X, y_{s_{1}}\right), \ldots, G\left(X, y_{s_{n}}\right) \mathbf{1}_{\left[0, \tau_{n}^{p(X)}\right]}(u) \frac{<p \cdot(X), W>_{u}}{p_{u}(X)}\right) d u\right. \\
& =E\left(\mathbf{1}_{A} h\left(G\left(X, y_{s_{1}}\right), \ldots, G\left(X, y_{s_{n}}\right) \int_{s \wedge \tau_{n}^{p(X)}}^{t \wedge \tau_{n}^{p(X)}} \frac{<p .(X), W>_{u}}{p_{u}(X)} d u\right) .\right.
\end{aligned}
$$

Arguing now as in Proposition 3, we obtain that $W_{t}-\int_{0}^{t} \beta_{s} d s$ is a continuous local martingale in the filtration $\left(\mathcal{H}_{t}\right)_{t \in[0, T[}$. Lévy's characterization theorem and the condition $\int_{0}^{T}\left|\beta_{s}\right| d s<\infty$ a.s. imply the result.

The following proposition has an appropriate form to apply Malliavin's Calculus in the computation of $\beta$.

Proposition 6 Suppose that $X$ is an $\mathcal{F}_{T}$-measurable random variable. Assume that there exists a $\mathcal{B}_{[0, T]} \otimes \mathcal{F}_{T}$-measurable process $\xi=\left\{\xi_{t}, t \in[0, T]\right\}$ such that $\int_{0}^{T} E\left(\left|\xi_{t}\right|\right) d t<\infty$, and that for any measurable and bounded function $f$ we have

$$
\begin{gathered}
f(X)=E(f(X))+\int_{0}^{T} \Phi_{t}^{f} d W_{t} \\
\Phi_{t}^{f}=E\left(f(X) \xi_{t} \mid \mathcal{F}_{t}\right),
\end{gathered}
$$

for almost all $(t, \omega)$. Then, $X$ belongs to the class $\mathcal{L}_{1}$, the signed measure $P_{t}^{(1)}(d x)$ is absolutely continuous with respect to $P_{t}(d x)$ for almost all $(t, \omega)$, and the density $\alpha_{t}(x)=\frac{P_{t}^{(1)}(d x)}{P_{t}(d x)}$ satisfies

$$
\alpha_{t}(X)=E\left(\xi_{t} \mid \mathcal{F}_{t} \vee \sigma(X)\right)
$$


for almost all $(t, \omega)$. Moreover,

$$
\beta_{t}:=E\left(\alpha_{t}(X) \mid \mathcal{H}_{t}\right)=E\left(\xi_{t} \mid \mathcal{H}_{t}\right)
$$

Proof. Any $\mathcal{F}_{t} \vee \sigma(X)$-measurable application from $\Omega$ to $\mathbb{R}$ factorizes in the form $\alpha_{t}(\omega, X(\omega))$ for an appropriate $\mathcal{F}_{t} \otimes \mathcal{B}(\mathbb{R})$-measurable application $\alpha_{t}(\omega, x): \Omega \times \mathbb{R} \rightarrow \mathbb{R}$. Let $\alpha_{t}(\omega, x)$ be the $\mathcal{F}_{t} \otimes \mathcal{B}(\mathbb{R})$-measurable application that verifies

$$
\alpha_{t}(X)=E\left(\xi_{t} \mid \mathcal{F}_{t} \vee \sigma(X)\right)
$$

where we omit the explicit dependence on $\omega$. As in Proposition 3 we can choose a version of $\alpha_{t}(x) \mathcal{P} \otimes \mathcal{B}(\mathbb{R})$-measurable, where $\mathcal{P}$ denotes the progressive $\sigma$-field. Setting $P_{t}^{(1)}(d x)=\alpha_{t}(x) P_{t}(d x)$ we obtain that $X$ belongs to the class $\mathcal{L}_{1}$. In fact,

$$
\begin{aligned}
E\left(f(X) \int_{0}^{t} u_{t} d W_{t}\right) & =E\left(\int_{0}^{t} E\left(f(X) \xi_{t} \mid \mathcal{F}_{t}\right) u_{t} d t\right) \\
& =E \int_{0}^{t} E\left(E\left(f(X) \xi_{t} \mid \mathcal{F}_{t} \vee \sigma(X)\right) \mid \mathcal{F}_{t}\right) u_{t} d t \\
& =E \int_{0}^{t} E\left(f(X) \alpha_{t}(X) \mid \mathcal{F}_{t}\right) u_{t} d t \\
& =\int_{0}^{t} E\left(\int_{\mathbb{R}} f(x) u_{t} \alpha_{t}(x) P_{t}(d x)\right) d t
\end{aligned}
$$

Notice that so far the values of $\alpha$ are independent of the process $Y$. Finally, in order to show (3), fix $t \in[0, T]$ such that $E\left(\left|\xi_{t}\right|\right)<\infty$ and take $B \in \mathcal{F}_{t}, h$ a bounded measurable function on $\mathbb{R}^{n}, s_{1} \leq \cdots \leq s_{n} \leq t$ and set $H=h\left(L_{s_{1}}, \ldots, L_{s_{n}}\right)$. Then, using the same notation as in the proof of proposition 3, we have

$$
\begin{aligned}
& E_{Y}\left(\xi_{t} \mathbf{1}_{B} H\right) \\
= & E\left(\xi_{t} \mathbf{1}_{B} h\left(G\left(X, y_{s_{1}}\right), \ldots, G\left(X, y_{s_{n}}\right)\right)\right. \\
= & E\left(E\left(\xi_{t} \mid \mathcal{F}_{t} \vee \sigma(X)\right) \mathbf{1}_{B} h\left(G\left(X, y_{s_{1}}\right), \ldots, G\left(X, y_{s_{n}}\right)\right)\right. \\
= & E\left(\alpha_{t} \mathbf{1}_{B}(X) h\left(G\left(X, y_{s_{1}}\right), \ldots, G\left(X, y_{s_{n}}\right)\right)\right. \\
= & E_{Y}\left(\alpha_{t}(X) \mathbf{1}_{B} H\right) .
\end{aligned}
$$

This implies (3). 
In general (2) is obtained through an integration by parts as we shall see in the examples below. Therefore the process $\xi$ need not be $\mathcal{F}_{t}$-adapted.

The next proposition gives a general formula for $\beta$ in the case of additive noise. In the sequel we consider the particular case where $G(x, y)=x+y$ and $Y_{t}=Z_{T-t}, Z$ being a continuous process with independent increments whose marginal has density $q_{t}$.

Proposition 7 Suppose that the assumptions of Proposition 3 hold. Let for $t \in[0, T]$ the random variables $L_{t}$ be given by $L_{t}=X+Y_{t}$. Then we have for $t \in[0, T]$

$$
\beta_{t}=\frac{\int_{\mathbb{R}} \alpha_{t}(x) q_{T-t}\left(L_{t}-x\right) P_{t}(d x)}{\int_{\mathbb{R}} q_{T-t}\left(L_{t}-x\right) P_{t}(d x)}=\frac{\int_{\mathbb{R}} q_{T-t}\left(L_{t}-x\right) P_{t}^{(1)}(d x)}{\int_{\mathbb{R}} q_{T-t}\left(L_{t}-x\right) P_{t}(d x)} .
$$

Proof. For $t \in[0, T]$ we may write, using the independence of $\mathcal{F}_{T}$ and $Y$

$$
\begin{aligned}
\beta_{t} & =E\left(\alpha_{t}(X) \mid \mathcal{F}_{t} \vee \sigma\left(L_{s}: s \leq t\right)\right) \\
& =E\left(\alpha_{t}(X) \mid \mathcal{F}_{t} \vee \sigma\left(L_{t}\right) \vee \sigma\left(Y_{t}-Y_{s}: s \leq t\right)\right) \\
& =E\left(\alpha_{t}(X) \mid \mathcal{F}_{t} \vee \sigma\left(L_{t}\right)\right) .
\end{aligned}
$$

Let $Q_{t}$ be the regular conditional probability of $\left(X, X+Y_{t}\right)$ given $\mathcal{F}_{t}$. Then for $C \in \mathcal{B}\left(\mathbb{R}^{2}\right)$

$Q_{t}(C)=\int_{\mathbb{R}^{2}} \mathbf{1}_{C}(x, x+y) q_{T-t}(y) P_{t}(d x) d y=\int_{\mathbb{R}^{2}} \mathbf{1}_{C}(x, l) q_{T-t}(l-x) P_{t}(d x) d l$.

Hence for $A \in \mathcal{B}(\mathbb{R})$

$$
P\left(X \in A \mid \mathcal{F}_{t} \vee \sigma\left(L_{t}\right)\right)=\frac{\int_{A} q_{T-t}\left(L_{t}-x\right) P_{t}(d x)}{\int_{\mathbb{R}} q_{T-t}\left(L_{t}-x\right) P_{t}(d x)} .
$$

Using $\alpha_{t}(x)=\frac{P_{t}^{(1)}(d x)}{P_{t}(d x)}$, we obtain

$$
\begin{aligned}
E\left(\alpha_{t}(X) \mid \mathcal{F}_{t} \vee \sigma\left(L_{t}\right)\right) & =\frac{\int_{\mathbb{R}} \alpha_{t}(x) q_{T-t}\left(L_{t}-x\right) P_{t}(d x)}{\int_{\mathbb{R}} q_{T-t}\left(L_{t}-x\right) P_{t}(d x)} \\
& =\frac{\int_{\mathbb{R}} q_{T-t}\left(L_{t}-x\right) P_{t}^{(1)}(d x)}{\int_{\mathbb{R}} q_{T-t}\left(L_{t}-x\right) P_{t}(d x)} .
\end{aligned}
$$




\section{$3 \quad$ Examples}

In the following examples we will treat particular cases. We will progressively specify the characteristics of the model trying to stay as general as possible. In every example we will compute the compensator $\beta_{t}=E\left(\alpha_{t}(X) \mid \mathcal{H}_{t}\right)$.

Example 1 Let $L_{t}=X+\tilde{W}_{g(T-t)}$, where $X=F\left(W_{T}\right), F: \mathbb{R} \rightarrow \mathbb{R}$ is a continuously differentiable function and $g:[0, T] \rightarrow[0,+\infty)$ is a strictly increasing bounded function with $g(0)=0$. Note that we cannot apply Proposition 7 directly since $F$ is not necessarily invertible. Assume that $E\left(F^{\prime}\left(W_{T}\right)^{2}\right)<\infty$. This is true, for example, if $\left|F^{\prime}(x)\right| \leq c e^{c x^{2}}$ and $c<1 / T$. For any smooth function $f$ with compact support we have

$$
\begin{aligned}
E\left(D_{t}\left(f\left(F\left(W_{T}\right)\right)\right) \mid \mathcal{F}_{t}\right) & =E\left(f^{\prime}\left(F\left(W_{T}\right)\right) F^{\prime}\left(W_{T}\right) \mid \mathcal{F}_{t}\right) \\
& =\int_{\mathbb{R}}(f \circ F)^{\prime}\left(W_{t}+x\right) \phi_{T-t}(x) d x,
\end{aligned}
$$

where $\phi_{t}$ denotes the Gaussian density with variance $t$. Integrating by parts we get

$$
\begin{aligned}
E\left(D_{t}\left(f\left(F\left(W_{T}\right)\right)\right) \mid \mathcal{F}_{t}\right) & =\int_{\mathbb{R}}(f \circ F)\left(W_{t}+x\right) \frac{x}{T-s} \phi_{T-t}(x) d x \\
& =E\left(f\left(F\left(W_{T}\right)\right) \frac{W_{T}-W_{t}}{T-t} \mid \mathcal{F}_{t}\right) .
\end{aligned}
$$

Hence, condition (2) of Proposition 6 is satisfied with $\xi_{t}=\frac{W_{T}-W_{t}}{T-t}$. This implies that

$$
\beta_{t}=E\left(\frac{W_{T}-W_{t}}{T-t} \mid \mathcal{H}_{t}\right)
$$

For $t \in[0, T]$ we may write, using the conditional independence of $\left\{W_{r}, r<\right.$ $s\}$ and $\sigma\left(L_{r}, r \leq s\right)$ given $W_{s}$ and the independence of $W$ and $\widetilde{W}$

$$
\begin{aligned}
E\left(\frac{W_{T}-W_{t}}{T-t} \mid \mathcal{H}_{t}\right) & =E\left(\frac{W_{T}-W_{t}}{T-t} \mid W_{t}, \sigma\left(L_{s}: s \leq t\right)\right) \\
& =E\left(\frac{W_{T}-W_{t}}{T-t} \mid W_{t}, F\left(W_{T}\right)+Y_{t}\right)
\end{aligned}
$$


where $Y_{t}=\tilde{W}_{g(T-t)}$. Let $Q_{t}$ be the regular conditional probability of $\left(W_{T}-\right.$ $\left.W_{t}, F\left(W_{T}\right)+Y_{t}\right)$ given $W_{t}=x$. Then for $C \in \mathcal{B}\left(\mathbb{R}^{2}\right)$

$$
\begin{aligned}
Q_{t}(C) & =\int_{\mathbb{R}^{2}} \mathbf{1}_{C}(y, F(x+y)+z) \phi_{g(T-t)}(z) \phi_{T-t}(y) d z d y \\
& =\int_{\mathbb{R}^{2}} \mathbf{1}_{C}(y, w) \phi_{g(T-t)}(w-F(x+y)) \phi_{T-t}(y) d w d y
\end{aligned}
$$

Hence for $A \in \mathcal{B}(\mathbb{R}), t<T$

$$
P\left(W_{T}-W_{t} \in A \mid W_{t}, L_{t}\right)=\frac{\int_{A} \phi_{g(T-t)}\left(L_{t}-F\left(W_{t}+y\right)\right) \phi_{T-t}(y) d y}{\int_{\mathbb{R}} \phi_{g(T-t)}\left(L_{t}-F\left(W_{t}+y\right)\right) \phi_{T-t}(y) d y}
$$

and

$$
\beta_{t}=\frac{\int_{\mathbb{R}} y \phi_{g(T-t)}\left(L_{t}-F\left(W_{t}+y\right)\right) \phi_{T-t}(y) d y}{(T-t) \int_{\mathbb{R}} \phi_{g(T-t)}\left(L_{t}-F\left(W_{t}+y\right)\right) \phi_{T-t}(y) d y} .
$$

Notice that $\frac{y}{T-t} \phi_{T-t}(y)=-\phi_{T-t}^{\prime}(y)$. Hence, integrating by parts yields

$$
\begin{aligned}
\beta_{t} & =\frac{\int_{\mathbb{R}}\left(L_{t}-F\left(W_{t}+y\right)\right) F^{\prime}\left(W_{t}+y\right) \phi_{g(T-t)}\left(L_{t}-F\left(W_{t}+y\right)\right) \phi_{T-t}(y) d y}{g(T-t) \int_{\mathbb{R}} \phi_{g(T-t)}\left(L_{t}-F\left(W_{t}+y\right)\right) \phi_{T-t}(y) d y} \\
& =\frac{1}{g(T-t)} E\left(Y_{t} F^{\prime}\left(W_{T}\right) \mid W_{t}, F\left(W_{T}\right)+Y_{t}\right) .
\end{aligned}
$$

In the particular case where $F$ is affine, i.e., $F(x)=\lambda x+a, \lambda \neq 0$, with $a \in \mathbb{R}$, we have

$$
\begin{aligned}
\beta_{t} & =\frac{\lambda}{g(T-t)} E\left(Y_{t} \mid W_{t}, \lambda W_{T}+a+Y_{t}\right) \\
& =\frac{\lambda}{g(T-t)} E\left(Y_{t} \mid \lambda\left(W_{T}-W_{t}\right)+Y_{t}\right) \\
& =\frac{\left(\lambda\left(W_{T}-W_{t}\right)+Y_{t}\right) \lambda}{g(T-t)+(T-t) \lambda^{2}} .
\end{aligned}
$$

Example 2. Let $X=M=\max _{0 \leq t \leq T} W_{t}, F(x, y)=x+y, Y_{t}=\tilde{W}_{g(T-t)}$ and $L_{t}=M+\tilde{W}_{g(T-t)}, t \in[0, T]$. For abbreviation, set $M_{t}=\max _{0 \leq s \leq t} W_{s}$, $t \in[0, T]$, and

$$
\beta_{t, T}=\max _{t \leq s \leq T}\left(W_{s}-W_{t}\right)
$$


Then

$$
M=M_{t} \vee\left(\beta_{t, T}+W_{t}\right)
$$

For any bounded and measurable function $f$ on $\mathbb{R}$ we can write

$$
\begin{aligned}
f(M) & =f(M)\left(\mathbf{1}_{\left\{M=M_{t}\right\}}+\mathbf{1}_{\left\{M>M_{t}\right\}}\right) \\
& =f\left(M_{t}\right) \mathbf{1}_{\left\{M=M_{t}\right\}}+f\left(\beta_{t, T}+W_{t}\right) \mathbf{1}_{\left\{\beta_{t, T}+W_{t}>M_{t}\right\}} .
\end{aligned}
$$

From this decomposition we can find a regular version of the conditional law of $M$ given $\mathcal{F}_{t}, t \in[0, T]$. Indeed,

$$
\begin{aligned}
E\left(f(M) \mid \mathcal{F}_{t}\right) & =E\left(f\left(M_{t}\right) \mathbf{1}_{\left\{M=M_{t}\right\}}+f\left(\beta_{t, T}+W_{t}\right) \mathbf{1}_{\left\{\beta_{t, T}+W_{t}>M_{t}\right\}} \mid \mathcal{F}_{t}\right) \\
& =f\left(M_{t}\right) R_{T-t}\left(M_{t}-W_{t}\right)+\int_{M_{t}-W_{t}}^{\infty} f\left(x+W_{t}\right) r_{T-t}(x) d x
\end{aligned}
$$

where $r_{t}$ and $R_{t}$ denote the density and the distribution function, respectively, of the maximum of the Wiener process in the interval $[0, t]$, which are given by

$$
r_{t}(x)=\sqrt{\frac{2}{\pi t}} \exp \left(-\frac{x^{2}}{2 t}\right), \quad x>0
$$

and

$$
R_{t}(y)=\int_{0}^{y} r_{t}(x) d x, \quad y \geq 0
$$

Hence,

$$
P_{t}(d x)=\delta_{M_{t}}(d x) R_{T-t}\left(M_{t}-W_{t}\right)+r_{T-t}\left(x-W_{t}\right) \mathbf{1}_{\left(M_{t}, \infty\right)}(x) d x .
$$

On the other hand, one can show that

$$
P_{t}^{(1)}(d x)=-\delta_{M_{t}}(d x) r_{T-t}\left(M_{t}-W_{t}\right)+\frac{x-W_{t}}{T-t} r_{T-t}\left(x-W_{t}\right) \mathbf{1}_{\left(M_{t}, \infty\right)}(x) d x
$$


In fact, by Clark-Ocone's formula we obtain for any smooth function $f$ with compact support

$$
\begin{aligned}
E\left[D_{t}(f(M)) \mid \mathcal{F}_{t}\right] & =E\left[f^{\prime}(M) D_{t} M \mid \mathcal{F}_{t}\right]=E\left[f^{\prime}(M) \mathbf{1}_{\left\{M>M_{t}\right\}} \mid \mathcal{F}_{t}\right] \\
& =E\left[f^{\prime}\left(\beta_{t, T}+W_{t}\right) \mathbf{1}_{\left\{\beta_{t, T}+W_{t}>M_{t}\right\}} \mid \mathcal{F}_{t}\right] \\
& =\int_{M_{t}-W_{t}}^{\infty} f^{\prime}\left(x+W_{t}\right) r_{T-t}(x) d x
\end{aligned}
$$

Integrating by parts yields

$$
\begin{aligned}
E\left[D_{t}(f(M)) \mid \mathcal{F}_{t}\right]= & -f\left(M_{t}\right) r_{T-t}\left(M_{t}-W_{t}\right) \\
& +\int_{M_{t}-W_{t}}^{\infty} f\left(x+W_{t}\right) \frac{x}{T-t} r_{T-t}(x) d x,
\end{aligned}
$$

which implies (7). Therefore by application of Proposition 6 one obtains that $W$ is a $\mathcal{H}_{t}$-semimartingale, $t \in[0, T]$, and that

$$
\alpha_{t}(x)=-\frac{r_{T-t}\left(M_{t}-W_{t}\right)}{R_{T-t}\left(M_{t}-W_{t}\right)} 1\left(x=M_{t}\right)+\frac{x-W_{t}}{T-t} \mathbf{1}_{\left(M_{t}, \infty\right)}(x) .
$$

From these formulas, Proposition 7 allows to deduce the following representation of $\beta$. We obtain for $t \in[0, T]$

$$
\beta_{t}=\frac{-r_{T-t}\left(M_{t}-W_{t}\right) q_{T-t}\left(L_{t}-M_{t}\right)+\int_{M_{t}}^{\infty} r_{T-t}\left(x-W_{t}\right) \frac{x-W_{t}}{T-t} q_{T-t}\left(L_{t}-x\right) d x}{R_{T-t}\left(M_{t}-W_{t}\right) q_{T-t}\left(L_{t}-M_{t}\right)+\int_{M_{t}}^{\infty} r_{T-t}\left(x-W_{t}\right) q_{T-t}\left(L_{t}-x\right) d x} .
$$

Now similar techniques as in the previous example will be used in order to assess the integrability properties of $\beta$. First of all, we note that

$$
r_{T-t}\left(x-W_{t}\right) \frac{x-W_{t}}{T-t}=-\frac{\partial}{\partial x} r_{T-t}\left(x-W_{t}\right), \quad x>W_{t}
$$

We use this formula to integrate by parts the second expression in the numerator of the representation of $\beta_{t}$. The result obviously is

$$
\begin{aligned}
\int_{M_{t}}^{\infty} & r_{T-t}\left(x-W_{t}\right) \frac{x-W_{t}}{T-t} q_{T-t}\left(L_{t}-x\right) d x \\
= & r_{T-t}\left(M_{t}-W_{t}\right) q_{T-t}\left(L_{t}-M_{t}\right) \\
& \quad+\frac{1}{g(T-t)} \int_{M_{t}}^{\infty} r_{T-t}\left(x-W_{t}\right)\left(L_{t}-x\right) q_{T-t}\left(L_{t}-x\right) d x .
\end{aligned}
$$


Substituting this in (8) gives the alternative representation

$$
\beta_{t}=\frac{1}{g(T-t)} \frac{\int_{M_{t}}^{\infty} r_{T-t}\left(x-W_{t}\right)\left(L_{t}-x\right) q_{T-t}\left(L_{t}-x\right) d x}{R_{T-t}\left(M_{t}-W_{t}\right) q_{T-t}\left(L_{t}-M_{t}\right)+\int_{M_{t}}^{\infty} r_{T-t}\left(x-W_{t}\right) q_{T-t}\left(L_{t}-x\right) d x} .
$$

Then from (4) and (6) we have that

$$
\beta_{t}=\frac{1}{g(T-t)} E\left(Y_{t} \mathbf{1}_{\left\{M>M_{t}\right\}} \mid \mathcal{H}_{t}\right)=\frac{1}{g(T-t)} E\left(Y_{t} \mathbf{1}_{\left\{M>M_{t}\right\}} \mid \mathcal{F}_{t} \vee \sigma\left(L_{t}\right)\right) \text {. }
$$

Example 3. Let $\left(X_{t}\right)_{t \in[0, T]}$ be a one dimensional time homogeneous Markov process with transition density $p_{t}(x, y), x, y \in \mathbb{R}, t \in[0, T]$, determined by a stochastic differential equation

$$
d X_{t}=b\left(X_{t}\right) d t+\sigma\left(X_{t}\right) d W_{t} .
$$

Assume that the density function is continuously differentiable in $x$ and $y$ and that there is a function $\gamma_{t}(y, x), x, y \in \mathbb{R}$, which is also continuously differentiable in $x$ and $y$ such that we have

$$
\frac{\partial}{\partial y} p_{t}(y, x)=\gamma_{t}(y, x) \frac{\partial}{\partial x} p_{t}(y, x), \quad x, y \in \mathbb{R}, t \in(0, T) .
$$

Let then $X=X_{T}$, the final value of the Markov process. Let $L_{t}=X+$ $\tilde{W}_{g(T-t)}, t \in[0, T]$, and use the notations of Example 1. This time the Markov property yields for $A \in \mathcal{B}(\mathbb{R}), t \in[0, T]$ the equation

$$
P\left(X \in A \mid \mathcal{F}_{t}\right)=\int_{A} p_{T-t}\left(X_{t}, x\right) d x
$$

whence the conditional density of $X$ given $\mathcal{F}_{t}$ is given by

$$
P_{t}(d x)=p_{T-t}\left(X_{t}, x\right) d x, \quad x \in \mathbb{R} .
$$

Now we compute the signed measure $P_{t}^{(1)}(d x)$ by Proposition 6 . In fact,

$$
\begin{aligned}
\Phi_{t}^{f} & =E\left(D_{t} f\left(X_{T}\right) \mid \mathcal{F}_{t}\right)=D_{t} E\left(f\left(X_{T}\right) \mid \mathcal{F}_{t}\right) \\
& =D_{t} \int_{\mathbb{R}} f(x) p_{T-t}\left(X_{t}, x\right) d x \\
& =\int_{\mathbb{R}} f(x) D_{t} p_{T-t}\left(X_{t}, x\right) d x \\
& =\int_{\mathbb{R}} f(x) \sigma\left(X_{t}\right) \gamma_{t}\left(X_{t}, x\right) \frac{\partial}{\partial x} p_{T-t}\left(X_{t}, x\right) d x .
\end{aligned}
$$


Therefore

$$
P_{t}^{(1)}(d x)=\sigma\left(X_{t}\right) \gamma_{t}\left(X_{t}, x\right) \frac{\partial}{\partial y} p_{T-t}\left(X_{t}, x\right) d x .
$$

One can use Proposition 7 to obtain the compensator $\beta$. First we use integration by parts to compute the numerator

$$
\begin{aligned}
& \int_{\mathbb{R}} q_{T-t}\left(L_{t}-x\right) P_{t}^{(1)}(d x) \\
& \quad=\sigma\left(X_{t}\right) \int_{\mathbb{R}} q_{T-t}\left(L_{t}-x\right) \gamma_{t}\left(X_{t}, x\right) \frac{\partial}{\partial x} p_{T-t}\left(X_{t}, x\right) d x \\
& \quad=\sigma\left(X_{t}\right) \int_{\mathbb{R}} p_{T-t}\left(X_{t}, x\right)\left[\frac{\partial}{\partial x} \gamma_{t}\left(X_{t}, x\right)+\gamma_{t}\left(X_{t}, x\right) \frac{L_{t}-x}{g(T-t)}\right] q_{T-t}\left(L_{t}-x\right) d x \\
& \quad=\sigma\left(X_{t}\right) \int_{\mathbb{R}}\left[\frac{\partial}{\partial x} \gamma_{t}\left(X_{t}, x\right)+\gamma_{t}\left(X_{t}, x\right) \frac{L_{t}-x}{g(T-t)}\right] q_{T-t}\left(L_{t}-x\right) P_{t}(d x) .
\end{aligned}
$$

Hence,

$$
\beta_{t}=\frac{\sigma\left(X_{t}\right) \int_{\mathbb{R}}\left[\frac{\partial}{\partial x} \gamma_{t}\left(X_{t}, x\right)+\gamma_{t}\left(X_{t}, x\right) \frac{L_{t}-x}{g(T-t)}\right] q_{T-t}\left(L_{t}-x\right) P_{t}(d x)}{\int_{\mathbb{R}} q_{T-t}\left(L_{t}-x\right) P_{t}(d x)}
$$

\section{Additional utility and arbitrage possibili- ties}

Consider, for the sake of simplicity, a Black-Scholes model with one stock with risk $S_{t}$. That means, $S_{t}$ satisfies the stochastic differential equation

$$
d S_{t}=\mu S_{t} d t+\sigma S_{t} d W_{t}
$$

with some parameters $\sigma>0, \mu \in \mathbb{R}$ and initial condition $S_{0}$. We also consider a riskless stock $B_{t}=\exp \{r t\}$, where $r$ is the instantaneous interest rate.

If we take the logarithmic utility function and we try to maximize the expected utility of the terminal wealth of traders, for fixed initial wealth, the difference between regular traders $(\mathrm{R})$ and insiders $(\mathrm{I})$ is given by

$$
\max _{\pi \in \mathrm{I} \text {-portfolio }} E\left(\ln \left(\mathcal{W}_{T}^{\pi}\right)\right)-\max _{\pi \in \mathrm{R} \text {-portfolio }} E\left(\ln \left(\mathcal{W}_{T}^{\pi}\right)\right)
$$


where $\mathcal{W}_{T}^{\pi}$ is the value of the portfolio $\pi$, and $\mathrm{I}(\mathrm{R})$-portfolios are the sets of admissible portfolios for the insiders (regular traders). It can be shown by slightly extending the techniques developed in Amendinger et al. (1998) that this difference is given by

$$
\frac{1}{2} E\left(\int_{0}^{T} \beta_{t}^{2} d t\right),
$$

and that the optimal portfolio is such that the amount of money invested in the risky asset is given by

$$
\frac{\mu+\sigma \beta_{t}-r}{\sigma^{2}} \mathcal{W}_{T}^{\pi}
$$

In this section we also study possible arbitrage opportunities. As usual, we shall say that a portfolio $\pi$ is an arbitrage opportunity if $\mathcal{W}_{0}^{\pi}=0$ and $P\left\{\mathcal{W}_{T}^{\pi} \geq 0\right\}=1$ with $P\left\{\mathcal{W}_{T}^{\pi}>0\right\}>0$. To avoid "doubling strategies" we shall impose the condition $\mathcal{W}_{t}^{\pi} \geq C$ a.s. for some constant $C \in \mathbb{R}$ and for any $t \in[0, T]$, that is we only admit the so called tame portfolios.

In the insider filtration, $\mathcal{H}_{t}$, and under the conditions of the previous propositions, we can write

$$
d S_{t}=\left(\mu+\sigma \beta_{t}\right) S_{t} d t+\sigma S_{t} d W_{t}^{*}
$$

where $W_{t}^{*}=W_{t}-\int_{0}^{t} \beta_{s} d s$ is an $\mathcal{H}_{t}$-Brownian motion. Suppose that $\int_{0}^{T} \beta_{t}^{2} d t<$ $\infty$ a.s and that there exists a probability measure $Q^{*}$ equivalent to $P$ such that $W_{t}=W_{t}^{*}+\int_{0}^{t} \beta_{s} d s$ is an $\mathcal{H}_{t}$-Brownian motion. Now, again

$$
d S_{t}=\mu S_{t} d t+\sigma S_{t} d W_{t}
$$

but where $W_{t}$ is an $\mathcal{H}_{t}$-Brownian motion with respect to $Q^{*}$. It is obvious now that there exits a probability measure $Q$ equivalent to $Q^{*}$ such that

$$
d S_{t}=r S_{t} d t+\sigma S_{t} d \hat{W}_{t}
$$

where $\hat{W}_{t}$ is an $\mathcal{H}_{t}$-Brownian motion with respect to $Q$. By composing the two steps we have a probability measure $Q$ equivalent to $P$, such that

$$
\hat{W}_{t}=W_{t}^{*}+\int_{0}^{t}\left(\frac{\mu-r}{\sigma}+\beta_{s}\right) d s
$$


is an $\mathcal{H}_{t}$-Brownian motion with respect to $Q$. Then, according to Corollary 2 in Levental and Skorohod (1995) there will not be arbitrage opportunities with tame portfolios. So, we simply have to know whether or not there is a probability measure $Q^{*}$ equivalent to $P$ such that $W_{t}=W_{t}^{*}+\int_{0}^{t} \beta_{s} d s$ is an $\left(\mathcal{H}_{t}\right)$-Brownian motion with respect to $Q^{*}$.

Example 1 Suppose that $L_{t}=X+\tilde{W}_{g(T-t)}$, where $X=F\left(W_{T}\right)$, with $E\left(F^{\prime}\left(W_{T}\right)^{2}\right)<\infty$. We have shown that

$$
\beta_{t}=\frac{1}{g(T-t)} E\left(Y_{t} F^{\prime}\left(W_{T}\right) \mid W_{t}, F\left(W_{T}\right)+Y_{t}\right),
$$

where $Y_{t}=\tilde{W}_{g(T-t)}$. Hence, applying Cauchy-Schwarz's inequality we obtain

$$
E\left(\beta_{t}^{2}\right) \leq \frac{1}{g(T-t)^{2}} E\left(Y_{t}^{2} F^{\prime}\left(W_{T}\right)^{2}\right)=\frac{1}{g(T-t)} E\left(F^{\prime}\left(W_{T}\right)^{2}\right) .
$$

Therefore we conclude,

$$
E\left(\int_{0}^{T} \beta_{t}^{2} d t\right) \leq E\left(F^{\prime}\left(W_{T}\right)^{2}\right) \int_{0}^{T} \frac{d t}{g(t)},
$$

and $E\left(\int_{0}^{T} \beta_{t}^{2} d t\right)<\infty$ if $\int_{0}^{T} \frac{d t}{g(t)}<\infty$. This condition is satisfied, for instance, in the case $g(s)=K s^{p}$ with $0<p<1, K>0$.

Let us take

$$
X=\log \left(S_{T}\right)=\log S_{0}+\tilde{\mu} T+\sigma W_{T},
$$

with $\tilde{\mu}=\mu-\sigma^{2} / 2$. Then $F$ is a linear function and as a consequence,

$$
\beta_{t}=\frac{\sigma^{2}\left(W_{T}-W_{t}\right)+\sigma \tilde{W}_{g(T-t)}}{\sigma^{2}(T-t)+g(T-t)}=\frac{\sigma\left(L_{t}-\log \left(S_{t}\right)-\tilde{\mu}(T-t)\right)}{\sigma^{2}(T-t)+g(T-t)},
$$

and

$$
E \int_{0}^{T} \beta_{t}^{2} d t=\int_{0}^{T} \frac{\sigma^{2}}{\sigma^{2}(T-t)+g(T-t)} d t
$$

Since $\beta$ is a Gaussian proces $E\left(\int_{0}^{T} \beta_{t}^{2} d t\right)<\infty$ is a sufficient condition (see Lipster and Shiryaev (1997)) to guarantee the existence of $Q^{*}$, then if we take $g(s)=K s^{p}$ with $0<p<1$, there are no arbitrage opportunities. But 
if $g(s)=K s^{p}$ with $p \geq 1$ we have, by the law of the iterated logarithm and because $W$ and $\tilde{W}$ are independent, that

$$
\left(\frac{\sigma^{2}\left(W_{T}-W_{t}\right)+\sigma \tilde{W}_{g(T-t)}}{\sigma^{2}(T-t)+g(T-t)}\right)^{2}=0\left((T-t)^{-1} \log \log (1 /(T-t))\right) \quad \text { a.s., when } t \rightarrow T
$$

and since

$$
\begin{gathered}
\int_{0}^{T} t^{-1} \log \log (1 / t) d t=\infty \\
\int_{0}^{T} \beta_{t}^{2} d t=\infty \quad \text { a.s. }
\end{gathered}
$$

Levental and Skorohod (1995) have constructed an arbitrage opportunity for this situation as exhibited in the proof of Theorem 1 of their paper.

Moreover, note that if $0<p<1$,

$\frac{\sigma^{2}\left(W_{T}-W_{t}\right)+\sigma \tilde{W}_{g(T-t)}}{\sigma^{2}(T-t)+g(T-t)}=0\left((T-t)^{-p / 2} \sqrt{ }\left(\log \log \left(1 /(T-t)^{p}\right)\right)\right)$ a.s., when $t \rightarrow T$.

Then $\lim _{t \rightarrow T}\left|\beta_{t}\right|=\infty$ a.s and by (16), the insider becomes a large trader when the revelation time $T$ approaches.

Example 2 Suppose that $L_{t}=M+\tilde{W}_{g(T-t)}$, where $M=\max _{0 \leq t \leq T} W_{t}$, and set $M_{t}=\max _{0 \leq s \leq t} W_{s}$. We have shown that

$$
\left.\beta_{t}=\frac{1}{g(T-t)} E\left(Y_{t} \mathbf{1}_{\left\{M>M_{t}\right\}} \mid \mathcal{F}_{t} \vee \sigma\left(L_{t}\right)\right)\right)
$$

Applying Cauchy-Schwartz's inequality yields

$$
E\left(\beta_{t}^{2}\right) \leq \frac{1}{g(T-t)^{2}} E\left(Y_{t}^{2} \mathbf{1}_{\left\{M>M_{t}\right\}}\right) \leq \frac{1}{g(T-t)^{2}} E\left(Y_{t}^{2}\right)=\frac{1}{g(T-t)} .
$$

Again $E\left(\int_{0}^{T} \beta_{t}^{2} d t\right)<\infty$ if $\int_{0}^{T} \frac{d t}{g(t)}<\infty$. This condition is satisfied, for instance, in the case $g(s)=K s^{p}$ with $0<p<1, K>0$. 
A sufficient condition to guarantee the existence of $Q^{*}$ is the Novikov condition:

$$
E\left(\exp \left\{\frac{1}{2} \int_{0}^{T} \beta_{t}^{2} d t\right\}\right)<\infty
$$

In this case we have

$$
\begin{aligned}
\beta_{t}^{2} & \leq \frac{1}{g(T-t)^{2}} E\left(Y_{t}^{2} \mid \mathcal{F}_{t} \vee \sigma\left(L_{t}\right)\right) \\
& \leq \frac{1}{g(T-t)^{2}} E\left(\sup _{0 \leq t \leq T} Y_{t}^{2} \mid \mathcal{F}_{t} \vee \sigma\left(L_{t}\right)\right)
\end{aligned}
$$

then writing $U_{t}=E\left(\sup _{0 \leq t \leq T} Y_{t}^{2} \mid \mathcal{F}_{t} \vee \sigma\left(L_{t}\right)\right)$, and assuming that $\int_{0}^{T} \frac{1}{g(T-t)^{2}} d t<$ $\infty$ we have

$$
\begin{aligned}
& E\left(\exp \left\{\frac{1}{2} \int_{0}^{T} \beta_{t}^{2} d t\right\}\right) \\
\leq & E\left(\exp \left\{\frac{1}{2} \int_{0}^{T} \frac{U_{t}}{g(T-t)^{2}} d t\right\}\right) \\
\leq & E\left(\exp \left\{\frac{\sup _{0 \leq t \leq T} U_{t}}{2} \int_{0}^{T} \frac{1}{g(T-t)^{2}} d t\right\}\right) \\
\leq & E\left(\sup _{0 \leq t \leq T}\left(\exp \left\{\frac{U_{t}}{4} \int_{0}^{T} \frac{1}{g(T-t)^{2}} d t\right\}\right)^{2}\right) \\
\leq & 4 E\left(\exp \left\{\frac{U_{T}}{2} \int_{0}^{T} \frac{1}{g(T-t)^{2}} d t\right\}\right)<\infty
\end{aligned}
$$

since $U_{T}=E\left(\sup _{0 \leq t \leq T}\left(Y_{t}^{2}\right) \mid \mathcal{F}_{T} \vee \sigma\left(L_{T}\right)\right)=E\left(\sup _{0 \leq t \leq T}\left(Y_{t}^{2}\right) \mid \mathcal{F}_{T}\right)=E\left(\sup _{0 \leq t \leq T}\left(Y_{t}^{2}\right)\right)<$ $\infty$.

Note that $\int_{0}^{T} \frac{1}{g(T-t)^{2}} d t<\infty$ is satisfied, for instance, in case $g(s)=K s^{p}$ with $0<p<1 / 2, K>0$. In these cases there are no arbitrage opportunities.

Example 3. This example can be seen as a generalization of the previous ones, disregarding the case where the function $\mathrm{F}$ is not one-to-one in example 1. The stochastic equation (10) can be consider as a generalization of the Black-Scholes model, where we assume implicitily the existence of a riskless asset, $B(t)$ that evolves as 


$$
d B(t)=B(t) r(t) d t, \quad B(0)=1,
$$

where $r(t)$ is an $\mathcal{F}_{t}$-adapted process that represents the instantaneous interest rate. Suppose that

$$
\int_{0}^{T}\left(\frac{b\left(X_{t}\right)-r(t)}{\sigma\left(X_{t}\right)}\right)^{2} d t<\infty \quad \text { a.s. }
$$

then we could argue as before to study the arbitrage opportunities in the insider filtration, but we only shall consider the utility gain of the insider.

Let us next apply Cauchy-Schwarz's inequality to the result of the integration by parts appearing in (13) and integrate with respect to the conditional law of $L_{t}$ given $\mathcal{F}_{t}$. We obtain

$$
\begin{aligned}
E\left(\beta_{t}^{2}\right) & =\int_{\mathbf{R}} E\left(\frac{\left(\int_{\mathbf{R}} q_{T-t}(y-x) P_{t}^{(1)}(d x)\right)^{2}}{\int_{\mathbf{R}} q_{T-t}(y-x) P_{t}(d x)}\right) d y \\
& \leq E\left(\int_{\mathbf{R}} \int_{\mathbf{R}} \sigma\left(X_{t}\right)^{2}\left[\frac{\partial}{\partial x} \gamma_{t}\left(X_{t}, x\right)+\gamma_{t}\left(X_{t}, x\right) \frac{y-x}{g(T-t)}\right]^{2} q_{T-t}(y-x) P_{t}(d x) d y\right) \\
& =E\left(\sigma\left(X_{t}\right)^{2}\left[\frac{\partial}{\partial x} \gamma_{t}\left(X_{t}, X_{T}\right)+\gamma_{t}\left(X_{t}, X_{T}\right) \frac{Y_{t}}{g(T-t)}\right]^{2}\right) .
\end{aligned}
$$

To transform line 2 into line 3 , one has to recall the joint law of $\left(X_{T}, X_{T}+Y_{t}\right)$. Therefore we conclude

$$
E\left(\int_{0}^{T} \beta_{t}^{2} d t\right) \leq \int_{0}^{T} E\left(\sigma\left(X_{t}\right)^{2}\left[\frac{\partial}{\partial x} \gamma_{t}\left(X_{t}, X_{T}\right)+\gamma_{t}\left(X_{t}, X_{T}\right) \frac{Y_{t}}{g(T-t)}\right]^{2}\right) d t
$$

and therefore

$$
E\left(\int_{0}^{T} \beta_{t}^{2} d t\right)<\infty
$$

if

$$
g(s)=K s^{p} \text { with } 0<p<1, K>0
$$

and

$$
\sup _{0 \leq t \leq T} E\left(\sigma\left(X_{t}\right)^{2} \gamma_{t}\left(X_{t}, X_{T}\right)^{2}\right)<\infty, \quad \sup _{0 \leq t \leq T} E\left(\sigma\left(X_{t}\right)^{2} \frac{\partial}{\partial x} \gamma_{t}\left(X_{t}, X_{T}\right)^{2}\right)<\infty
$$




\section{References}

[1] Amendinger, J., Imkeller, P., Schweizer, M., 1998. Additional logaritmic utility of an insider. Stochastic Proc. Appl. 75 (1998) 263-286.

[2] Baudoin, F., 2001. Portfolio optimization associated with a weak information. Technical report, Université Pierre et Marie Curie, 2001.

[3] Back, K., 1992. Insider Trading in Continuous Time. Review of Financial Studies 5, 387-409.

[4] Grorud, A., Pointier, M., 1998. Insider Trading in a continuous Time Market Model. International Journal of Theoretical and Applied Finance $1,331-347$.

[5] Imkeller, P., 1996. Enlargement of the Wiener filtration by an absolutely continuous random variable via Malliavin's calculus. Probab. Th. Rel . Fields 106, 105-135.

[6] Imkeller, P., 1997. Enlargement of the Wiener filtration by a manifold valued random element via Malliavin's calculus. In Statistics and Control of Stochastic Processes. The Lipster Festschrift, Y.M. Kabanov, B.L. Rosovskii, A.N. Shiryaev (eds.), World Scientific, Singapore.

[7] Imkeller, P., Pontier, M., Weisz, F., 2001. Free lunch and arbitrage possibilities in a financial market with an insider. Stochastic Proc. Appl. 92, 103-130.

[8] Jacod, J., 1985. Grossissement initial, hypothèse (H'), et théorème de Girsanov. In : Grossissements de filtrations: exemples et applications. T Jeulin, M. Yor (eds.) Lect. Notes in Maths. 1118. Springer-Verlag. Berlin.

[9] Karatzas, I., Pikovsky, I., 1996. Anticipative portfolio optimization. Adv. Appl. Prob. 28, 1095-1122.

[10] Kyle, A., 1985. Continuous Auctions and Insider Trading. Econometrica $53,1315-1335$.

[11] Levental, S., Skorohod, A. V., 1995. A necessary and sufficient condition for absence of arbitrage with tame porfotlios. Ann. Appl. Probab. 5, 906925. 
[12] Liptser, R.S., Shiryaev, A.N., 1997. Statistics of Random Processes I. General Theory. Springer-Verlag. New York.

[13] Meyer, P. A., 1996. Probability and Potentials. Blaisdell Publishing Company, Waltham, Mass.

[14] Nualart, D. , 1995. The Malliavin Calculus and Related Topics. SpringerVerlag. Berlin.

[15] Rogers, L.C.G. and Williams, D.,1979. Diffusions, Markov Processes, and Martingales. John Wiley. New York.

[16] Protter, P., 1990. Stochastic Integration and Differential Equations. A New Approach. Springer-Verlag. New York.

[17] Séminaire de Calcul Stochastique 1982/83, Université Paris VI, 1985. Grossisements de fitrations: exemples et applications. T Jeulin, M. Yor (eds.). Lect. Notes in Maths. 1118. Springer-Velag. Berlin

[18] Yor, M. Grossissement de filtrations et absolue continuite de noyaux. In : Grossissements de filtrations: exemples et applications. T Jeulin, M. Yor (eds.) Lect. Notes in Maths. 1118. Springer-Verlag. Berlin. 